\title{
Assignment of Non-equivalent Methylene Protons of Poly(3-hydroxybutyrate)
}

\author{
Kunio HIKICHI ${ }^{\dagger}$ and Kenji OGURA* \\ Section of Structural Bio-Macromolecular Science, Division of Biological Sciences, \\ Graduate School of Sciences, Hokkaido University, Sapporo 060, Japan
}

(Received June 29, 1994)

\begin{abstract}
KEY WORDS NMR / Assignment / Methylene / Poly(3-hydroxybutyrate) / Long-Range ${ }^{13} \mathrm{C}-{ }^{1} \mathrm{H}$ Coupling /
\end{abstract}

Poly(3-hydroxybutyrate) is a biodegradable polyester which is produced by bacteria. ${ }^{1}$ There have been increasing interests on the property and structure of this polymer. ${ }^{1} \mathrm{H}$ and ${ }^{13} \mathrm{C}$ nuclear magnetic resonance spectroscopies have been used for the study of the structure. The most important problem in studying structure by means of NMR is to assign each resonance peak to each proton or carbon. The polymer has a methylene group of which two protons are non-equivalent. The stereospecific assignment of the two non-equivalent protons of the methylene is needed for studying the conformation of this polymer.

$$
\begin{gathered}
\mathrm{CH}_{3} \\
\mathrm{C}-\mathrm{CH}-\underset{\mathrm{H}}{\mathrm{H}}-\mathrm{C})_{n}
\end{gathered}
$$

In a previous paper, ${ }^{2}$ the non-equivalent protons of methylene of poly(3-hydroxybutyrate) have been assigned on the basis of the proton NOE between the methyl and the methylene. In this paper, we present an alternative method of assignment based on the three-bond coupling constant ${ }^{3} J_{\mathrm{C}-\mathrm{H}}$ between the methyl carbon and the methylene protons by the help of the previous population results.

Poly(3-hydroxybutyrate) of a molecular weight of 800,000 was dissolved in $\mathrm{CDCl}_{3}$ at a concentration of $0.04 \mathrm{~g} / \mathrm{ml}$. NMR spectra were obtained by JEOL JNM-A600 and JEOL JNM-EX400 spectrometers at $26^{\circ} \mathrm{C}$.

Kamiya, et al., ${ }^{2}$ observed the ${ }^{1} \mathrm{H}$ spectra to obtain vicinal proton coupling constants ${ }^{3} J_{\mathbf{H}-\mathbf{H}}$ between methine and methylene. The results were analyzed by the rotation isomer model. They found that the population of trans, gauche, and gauche prime $\left(P_{\mathrm{T}}, P_{\mathrm{G}}, P_{\mathrm{G}^{\prime}}\right)$ is either of $(0.58,0.42,0)$ or $(0.42,0.58,0)$, depending on the assignment of the two non-equivalent protons. We confirmed their results.

To obtain the three-bond coupling constant between the methyl carbon and methylene protons, we carried out the long-range ${ }^{13} \mathrm{C}$ $J$-resolved two-dimensional NMR spectros-
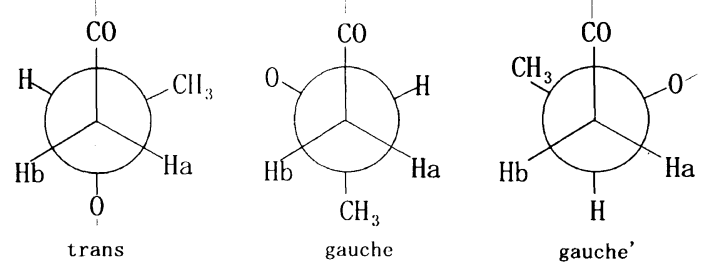

Figure 1. Figure 1. Newman Projections of rotation isomers.

$\dagger$ To whom correspondences should be addressed.

* Present address: Molecular Physics Department, Tokyo Metropolitan Institute of Medical Sciences, 3-18-22 Honkomagome, Bunkyoku, Tokyo 113, Japan. 
copy. ${ }^{3}$ It is found that ${ }^{3} J_{\mathrm{C}-\mathrm{H}}$ is $3.5 \mathrm{~Hz}$ for the high-field methylene proton and $4.7 \mathrm{~Hz}$ for the low-field methylene proton. These values are not accurate because of the imperfect selective proton irradiation to the methine proton, but we believe that the relative order of $J_{\text {low-field }}>$ $J_{\text {high-field }}$ is correct. We apply the rotasion isomer model to the observed three-bond coupling constant between the methyl carbon and the methylene protons.

$$
\begin{aligned}
& P_{\mathrm{T}} J_{\mathrm{GC}}+P_{\mathrm{G}} J_{\mathrm{GC}}+P_{\mathrm{G}^{\prime}} J_{\mathrm{TG}}=J_{\mathrm{C}-\mathrm{Ha}} \\
& P_{\mathrm{T}} J_{\mathrm{TC}}+P_{\mathrm{G}} J_{\mathrm{GC}}+P_{\mathrm{G}^{\prime}} J_{\mathrm{GC}}=J_{\mathrm{C}-\mathrm{Hb}} \\
& P_{\mathrm{T}}+P_{\mathrm{G}}+P_{\mathrm{G}^{\prime}}=1
\end{aligned}
$$

Because of $P_{\mathrm{G}^{\prime}}=0$, we obtain

$$
J_{\mathrm{TC}}-J_{\mathrm{GC}}=\left(J_{\mathrm{C}-\mathrm{Hb}}-J_{\mathrm{C}-\mathrm{Ha}}\right) / P_{\mathrm{T}}
$$

It is found that $J_{\mathrm{TC}}>J_{\mathrm{GC}} \cdot{ }^{4}$ Therefore, we can assign the high-field peak to $\mathrm{Ha}$ and the low- field peak to $\mathrm{Hb}$. This is in agreement with the previous results.

Acknowledgement. The authors are indebted to Professor Inoeu of Tokyo Institute of Technology for providing the sample used here. This work is partly supported by the Ministry of Education, Culture and Science of Japan.

\section{REFERENCES}

1. M. Lemoigne, Ann. Inst. Past., 10, 135 (1925).

2. Naoki Kamiya, Yoshio Inoue, Yasuhiko Yamamoto, Riichirô Chûjô, and Yoshiharu Doi, Macromolecules, 23, 1313 (1990).

3. H. Seto, JEOL NEWS, 24, 37 (1984).

4. J. L. Marshall, "Carbon-Carbon and CarbonProton NMR Couplings," Verlag Chemie International, DeerField Beach, Florida, 1983. 